\title{
Numerical Methods for Fractional-Order Fornberg-Whitham Equations in the Sense of Atangana-Baleanu Derivative
}

\author{
Naveed Iqbal $\mathbb{D}^{1}{ }^{1}$ Humaira Yasmin, ${ }^{2}$ Akbar Ali, ${ }^{1}$ Abdul Bariq $\left(D,{ }^{3}\right.$ M. Mossa Al-Sawalha, ${ }^{1}$ \\ and Wael W. Mohammed ${ }^{1,4}$ \\ ${ }^{1}$ Department of Mathematics, Faculty of Science, University of Ha'il, Ha'il 2440, Saudi Arabia \\ ${ }^{2}$ Department of Basic Sciences, Preparatory Year Deanship, King Faisal University, Al-Ahsa 31982, Saudi Arabia \\ ${ }^{3}$ Department of Mathematics, Laghman University, Mehterlam, 2701 Laghman, Afghanistan \\ ${ }^{4}$ Faculty of Science, Mansoura University, Mansoura 35516, Egypt
}

Correspondence should be addressed to Abdul Bariq; abdulbariq.maths@lu.edu.af

Received 3 October 2021; Revised 12 November 2021; Accepted 30 November 2021; Published 13 December 2021

Academic Editor: J. Vanterler da C. Sousa

Copyright (C) 2021 Naveed Iqbal et al. This is an open access article distributed under the Creative Commons Attribution License, which permits unrestricted use, distribution, and reproduction in any medium, provided the original work is properly cited.

In this paper, we investigate the numerical solution of the Fornberg-Whitham equations with the help of two powerful techniques: the modified decomposition technique and the modified variational iteration technique involving fractional-order derivatives with Mittag-Leffler kernel. To confirm and illustrate the accuracy of the proposed approach, we evaluated in terms of fractional order the projected models. Furthermore, the physical attitude of the results obtained has been acquired for the fractional-order different value graphs. The results demonstrated that the future method is easy to implement, highly methodical, and very effective in analyzing the behavior of complicated fractional-order linear and nonlinear differential equations existing in the related areas of applied science.

\section{Introduction}

The analysis of the Fornberg-Whitham equation (FWE) is a significant mathematical equation of mathematical physics. The Fornberg-Whitham equation is defined as $[1,2]$

$$
D_{\Im} \mu-D_{\zeta \zeta \Im} \mu+D_{\zeta} \mu=\mu D_{\zeta \zeta \zeta} \mu-\mu D_{\zeta} \mu+3 D_{\zeta} \mu D_{\zeta \zeta} \mu .
$$

This model was invented to evaluate the nonlinear breaking dispersive ocean waves. The Fornberg-Whitham equation is shown to yield peakon solutions as a physical equation for waves of restricting height and the occurrences of wave breaking. Fractional calculus is now widely used and accepted, owing to its well-known uses in a variety of fields of seemingly disparate sectors of science and engineering $[3,4]$. Many scholars, including Gupta and Singh [5] and Alderremy et al. [6], have examined the fractional of the Fornberg-Whitham equation relevant to the fractional Caputo derivative, Sunthrayuth et al. [7], Singh et al. [8], etc. Because of the singular kernel of the fractional Caputo derivative, its implementations are limited. Caputo and Fabrizio [9] created derivatives of any (real or complex) order with a nonsingular kernel. Caputo and Fabrizio's derivative has been used to a variety of real-world situations, including fractional nonhomogeneous heat models [10], El Nino-Southern fractional oscillations models [11], and arbitrary-order system of smoking models [12]. Atangana and Baleanu [13] devised a novel fractional-order derivative called the Atangana-Baleanu (AB) fractional derivative, which has the kernel of a Mittag-Leffler-type function. Kumar et al. [14] investigated the regularised long-wave equation with a Mittag-Leffler-type kernel incorporating the fractional operator. A Mittag-Leffler-type kernel is used in the chemical kinetics system connected with a fractional derivative which was investigated by Singh et al. [15]. Baleanu et al. [16] recently proposed optimal fractional models with nonsingular Mittag-Leffler kernels. As we all know, the Mittag-Leffler function is more beneficial in expressing physical difficulties than the power function or the exponential function; as a result, the $\mathrm{AB}$ fractional derivative is well suited to 
unravelling material heterogeneities and structures or media with different scales.

George Adomian was introduced to and established a technique for "solving integro-differential, differential equations, delay differential, and partial differential equations" $[17,18]$. The result is discovered as an infinite sequence that quickly converges to precise solution. This method has been shown to be effective in solving both linear and nonlinear models. The method for solving a nonlinear operator problem is to use decomposition equations in a series of functions. Each expression's sequence is derived from a polynomial derived from the expansion of an approximate solution into power sequences. The Adomian decomposition method technique is really simple in theory, but the difficulty arises when it comes to determining polynomials and illustrating the convergence of a series of functions [19]. Lesnic [20] analyzed the convergent of the Adomian decomposition method when using heat and wave models for both backward and forward time evolution. Gaber and El-Sayed used the Adomian method of solving fractal-order partial differential equations on a finite domain in [21]. Ghoreishi et al. [22] investigated the Adomian decomposition method's ability to investigate nonlinear wave problems with changing coefficients, demonstrating that the Adomian decomposition method can solve these equations without the need for dissertation, linearization, transformation, or perturbation.

The variational iteration approach $[23,24]$ was published in the late 1990s to solve a seepage flow with fractional derivatives and a nonlinear oscillator, and it has since been widely utilized as a primary analytical tool for solving a variety of nonlinear problems. It has fully grown into a fully fledged mathematical approach as a result of considerable research by a number of authors, including $\mathrm{He}[25,26]$, Ganji and Sadighi [27], Ozis and Yildirim [28], and Noor and Mohyud-Din [29]. On November 24, 2018, we searched Clarivate's Web of Science for "variational iteration approach" and got 3761 hits. The technique's identification of the Lagrange multiplier necessitates the understanding of variational theory [30], and the technique's sophisticated identification process may limit its implementation to realworld issues.

\section{Preliminary Concepts}

Definition 1. The Caputo fractional derivative is given as [31]

${ }_{0}^{C} \mathscr{D}_{\mathfrak{S}}^{\gamma} f(\zeta, \mathfrak{J})=\frac{1}{\Gamma(n-\gamma)} \int_{0}^{\mathfrak{J}}(\mathfrak{I}-\theta)^{n-\gamma-1} f^{(n)}(\zeta, \theta) \mathrm{d} \theta, \quad n-1<\gamma \leq n$.

Definition 2. The Laplace transformation connected with fractional Caputo derivative ${ }^{L C} D_{\mathfrak{J}}^{\gamma}\{f(\mathfrak{I})\}$ is expressed by [31]

$$
\mathbb{L}\left[{ }^{L C} D_{\mathfrak{J}}^{\gamma}\{f(\mathfrak{J})\}\right](s)=s^{\gamma} \mathbb{L}[f(x, \mathfrak{I})](s)-s^{\gamma-1} f(x, 0) .
$$

Definition 3. In Caputo sense, the Atangana-Baleanu derivative is defined as [31]

$$
{ }^{A B C} D_{\Im}^{\gamma}\{f(\mathfrak{I})\}=\frac{A(\gamma)}{1-\gamma} \int_{a}^{\mathfrak{J}} f^{\prime}(k) E_{\gamma}\left[-\frac{\gamma}{1-\gamma}(1-k)^{\gamma}\right] d k,
$$

where $A(\gamma)$ is a normalization function such that $A(0)=$ $A(1)=1, f \in H^{1}(a, b), b>a, \gamma \in[0,1]$, and $E_{\gamma}$ represent the Mittag-Leffler function.

Definition 4. The Atangana-Baleanu derivative in the Riemann-Liouville sense is defined as [31]

$$
{ }^{A B C} D_{\widetilde{J}}^{\gamma}\{f(\mathfrak{J})\}=\frac{A(\gamma)}{1-\gamma} \frac{d}{d \mathfrak{I}} \int_{a}^{\mathfrak{\Im}} f(k) E_{\gamma}\left[-\frac{\gamma}{1-\gamma}(1-k)^{\gamma}\right] d k .
$$

Definition 5. The Laplace transform connected with the Atangana-Baleanu operator is defined as [31]

$$
{ }^{A B} D_{\mathfrak{J}}^{\gamma}\{f(\mathfrak{I})\}(s)=\frac{A(\gamma) s^{\gamma} \mathbb{L}\{f(\mathfrak{I})\}(s)-s^{\gamma-1} f(0)}{(1-\gamma)\left(s^{\gamma}+(\gamma /(1-\gamma))\right)} .
$$

Definition 6. Consider $0<\gamma<1$, and $f$ is a function of $\gamma$; then, the fractional-order integral operator of $\gamma$ is given as [31]

${ }^{A B C} I_{\mathfrak{J}}^{\gamma}\{f(\mathfrak{I})\}=\frac{1-\gamma}{A(\gamma)} f(\mathfrak{I})+\frac{\gamma}{A(\gamma) \Gamma(\gamma)} \int_{a}^{\mathfrak{I}} f(k)(\mathfrak{I}-k)^{\gamma-1} d k$.

\section{The Methodology of Variational Iteration Method}

This section introduces the solution of fractional partial differential equations with the help of the variational iteration method.

$$
{ }^{A B C} D_{\widetilde{J}}^{\gamma} v(\zeta, \mathfrak{J})+\overline{\mathscr{G}}(\zeta, \mathfrak{I})+\mathcal{N}(\zeta, \mathfrak{I})-\mathscr{P}(\zeta, \mathfrak{I})=0, \quad \phi-1<\gamma \leq \phi .
$$

The initial condition is

$$
v(\zeta, 0)=g(\zeta)
$$

where ${ }^{A B C} D_{\Im}^{\gamma}=\partial^{\gamma} / \partial \mathfrak{J}^{\gamma}$ is the fractional derivative Caputo order $\gamma, \mathscr{G}$ and $\mathcal{N}$ are linear and nonlinear terms, respectively, and $\mathscr{P}$ is the source function.

The Laplace transformation is applied to equation (8); we get

$$
L\left[{ }^{A B C} D_{\mathfrak{J}}^{\gamma} v(\zeta, \mathfrak{I})\right]+L[\overline{\mathscr{G}}(\zeta, \mathfrak{I})+\mathcal{N}(\zeta, \mathfrak{J})-\mathscr{P}(\zeta, \mathfrak{I})]=0
$$


The Lagrange multiplier iterative method is

$$
L\left[{ }^{A B C} D_{\mathfrak{J}}^{\gamma} \mathcal{v}(\zeta, \mathfrak{\Im})\right]+L[\overline{\mathscr{G}}(\zeta, \mathfrak{\Im})+\mathcal{N}(\zeta, \mathfrak{J})-\mathscr{P}(\zeta, \mathfrak{I})]=0
$$

A Lagrange multiplier is as

$$
\lambda(s)=-\frac{\left(s^{\gamma}(1-\gamma)+\gamma\right)}{s^{\gamma}}
$$

Applying inverse Laplace transform $L^{-1}$, equation (11) can be written as

$$
\begin{aligned}
v_{\phi+1}(\zeta, \mathfrak{J})= & v_{\phi}(\zeta, \mathfrak{I})-L^{-1}\left[\frac{\left(s^{\gamma}(1-\gamma)+\gamma\right)}{s^{\gamma}}[-L\{\overline{\mathscr{G}}(\zeta, \mathfrak{I})\right. \\
& +\mathcal{N}(\zeta, \mathfrak{I})\}]-L[\mathscr{P}(\zeta, \mathfrak{I})]]
\end{aligned}
$$

\section{The Conceptualization of MDM}

In this section, we discuss the solution of fractional partial differential equations with the help of the modified decomposition method.

${ }^{A B C} D_{\mathfrak{J}}^{\gamma} v(\zeta, \mathfrak{I})+\overline{\mathscr{G}}(\zeta, \mathfrak{I})+\mathcal{N}(\zeta, \mathfrak{I})-\mathscr{P}(\zeta, \mathfrak{I})=0, \quad m-1<\gamma \leq m$.

The initial condition is

$$
v(\zeta, 0)=g(\zeta)
$$

where ${ }^{A B C} D_{\mathfrak{\Im}}^{\gamma}=\partial^{\gamma} / \partial \mathfrak{J}^{\gamma}$ is the fractional derivative of Caputo order $\gamma, \bar{G}$ and $\mathcal{N}$ are linear and nonlinear terms, respectively, and $\mathscr{P}$ is the source term.

Using Laplace transformation to equation (14), we get

$$
L\left[{ }^{A B C} D_{\mathfrak{J}}^{\gamma} \nu(\zeta, \mathfrak{I})\right]+L[\overline{\mathscr{G}}(\zeta, \mathfrak{I})+\mathcal{N}(\zeta, \mathfrak{I})-\mathscr{P}(\zeta, \mathfrak{I})]=0
$$

Taking the Laplace transform of differentiation property, we have

$$
\begin{aligned}
L[\nu(\zeta, \mathfrak{J})]= & \frac{1}{s} \nu(\zeta, 0)+\frac{\left(s^{\gamma}(1-\gamma)+\gamma\right)}{s^{\gamma}} L[\mathscr{P}(\zeta, \mathfrak{J})] \\
& \left.-\frac{\left(s^{\gamma}(1-\gamma)+\gamma\right)}{s^{\gamma}} L\{\overline{\mathscr{G}}(\zeta, \mathfrak{I})+\mathcal{N}(\zeta, \mathfrak{J})\}\right]
\end{aligned}
$$

MDM result of infinite series $v(\zeta, \mathfrak{\Im})$,

$$
v(\zeta, \mathfrak{J})=\sum_{\phi=0}^{\infty} v_{\phi}(\zeta, \mathfrak{\Im})
$$

$\mathcal{N}$ nonlinear function is defined as

$$
\mathcal{N}(\zeta, \mathfrak{\Im})=\sum_{\phi=0}^{\infty} \mathscr{A}_{\phi}
$$

The nonlinear terms can be analyzed with the aid of Adomian polynomials. So the Adomian polynomial formula is expressed as

$$
\mathscr{A}_{\phi}=\frac{1}{j !}\left[\frac{\partial^{\phi}}{\partial \lambda^{\phi}}\left\{\mathcal{N}\left(\sum_{\phi=0}^{\infty} \lambda^{\phi} v_{\phi}\right)\right\}\right]_{\lambda=0}
$$

Then, put equations (18) and (19) into (17), which gives

$$
\begin{aligned}
L\left[\sum_{\phi=0}^{\infty} \nu_{\phi}(\zeta, \mathfrak{I})\right]= & \frac{1}{s} \nu(\zeta, 0)+\frac{\left(s^{\gamma}(1-\gamma)+\gamma\right)}{s^{\gamma}} L\{\mathscr{P}(\zeta, \mathfrak{I})\} \\
& -\frac{\left(s^{\gamma}(1-\gamma)+\gamma\right)}{s^{\gamma}} L\left\{\overline{\mathscr{G}}\left(\sum_{\phi=0}^{\infty} v_{\phi}\right)+\sum_{\phi=0}^{\infty} \mathscr{A}_{\phi}\right\}
\end{aligned}
$$

Applying the inverse Laplace transformation to equation (21), we get

$$
\begin{aligned}
\sum_{\phi=0}^{\infty} v_{\phi}(\zeta, \mathfrak{I})= & L^{-1}\left[\frac{1}{s} \nu(\zeta, 0)+\frac{\left(s^{\gamma}(1-\gamma)+\gamma\right)}{s^{\gamma}} L\{\mathscr{P}(\zeta, \mathfrak{I})\}\right. \\
& \left.-\frac{\left(s^{\gamma}(1-\gamma)+\gamma\right)}{s^{\gamma}} L\left\{\overline{\mathscr{G}}\left(\sum_{\phi=0}^{\infty} \nu_{\phi}\right)+\sum_{\phi=0}^{\infty} \mathscr{A}_{\phi}\right\}\right]
\end{aligned}
$$

Define the terms as follows:

$$
\begin{aligned}
& v_{0}(\zeta, \mathfrak{J})=L^{-1}\left[\frac{1}{s} \nu(\zeta, 0)+\frac{\left(s^{\gamma}(1-\gamma)+\gamma\right)}{s^{\gamma}} L\{\mathscr{P}(\zeta, \mathfrak{\Im})\}\right] \\
& v_{1}(\zeta, \mathfrak{J})=-L^{-1}\left[\frac{\left(s^{\gamma}(1-\gamma)+\gamma\right)}{s^{\gamma}} L\left\{\overline{\mathscr{G}}_{1}\left(\nu_{0}\right)+\mathscr{A}_{0}\right\}\right] .
\end{aligned}
$$

In general, $\phi \geq 1$ is defined as

$$
v_{\phi+1}(\zeta, \mathfrak{\Im})=-L^{-1}\left[\frac{\left(s^{\gamma}(1-\gamma)+\gamma\right)}{s^{\gamma}} L\left\{\overline{\mathscr{G}}\left(\nu_{\phi}\right)+\mathscr{A}_{\phi}\right\}\right]
$$

\section{Application of Techniques}

Example 7. Consider the time-fractional nonlinear Fornberg-Whitham equation

$$
D_{\mathfrak{J}}^{\gamma} v-D_{\zeta \zeta \Im} v+D_{\zeta} v=v D_{\zeta \zeta \zeta} v-v D_{\zeta} v+3 D_{\zeta} v D_{\zeta \zeta} v, \quad 0<\gamma \leq 1,
$$

with the initial condition 


$$
v(\zeta, 0)=e^{(\zeta / 2)}
$$

Taking Laplace transformation of (25),

$$
\begin{aligned}
& \frac{s^{\gamma}}{\left(s^{\gamma}(1-\gamma)+\gamma\right)}\left\{L[\nu(\zeta, \Im)]-\frac{1}{s} v(\zeta, 0)\right\} \\
& \quad=L\left[D_{\zeta \zeta \Im} v-D_{\zeta} v+v D_{\zeta \zeta \zeta} v-v D_{\zeta} v+3 D_{\zeta} v D_{\zeta \zeta} v\right] .
\end{aligned}
$$

Using inverse Laplace transformation

$$
\begin{aligned}
v(\zeta, \mathfrak{I})= & L^{-1}\left[\frac{v(\zeta, 0)}{s}-\frac{\left(s^{\gamma}(1-\gamma)+\gamma\right)}{s^{\gamma}} L\left[D_{\zeta \zeta \Im} v-D_{\zeta} v\right.\right. \\
& \left.\left.+v D_{\zeta \zeta \zeta} v-v D_{\zeta} v+3 D_{\zeta} v D_{\zeta \zeta} v\right]\right] .
\end{aligned}
$$

Applying Adomian procedure, we have

$$
\begin{aligned}
& v_{0}(\zeta, \mathfrak{J})=L^{-1}\left[\frac{\nu(\zeta, 0)}{s}\right]=L^{-1}\left[\frac{e^{(\zeta / 2)}}{s}\right] \text {, } \\
& v_{0}(\zeta, \mathfrak{I})=e^{(\zeta / 2)} \\
& \sum_{\phi=0}^{\infty} v_{\phi+1}(\zeta, \mathfrak{J})=L^{-1}\left[\frac { ( s ^ { \gamma } ( 1 - \gamma ) + \gamma ) } { s ^ { \gamma } } L \left[\sum_{\phi=0}^{\infty}\left(D_{\zeta \zeta \mathfrak{J}^{\nu}}\right)_{\phi}-\sum_{\phi=0}^{\infty}\left(D_{\zeta} v\right)_{\phi}\right.\right. \\
& \left.\left.+\sum_{\phi=0}^{\infty} A_{\phi}-\sum_{\phi=0}^{\infty} B_{\phi}+3 \sum_{\phi=0}^{\infty} C_{\phi}\right]\right], \quad \phi=0,1,2, \cdots, \\
& A_{0}\left(\nu D_{\zeta \zeta \zeta} v\right)=v_{0} D_{\zeta \zeta \zeta} v_{0} \\
& B_{0}\left(v D_{\zeta} v\right)=v_{0} D_{\zeta} v_{0} \\
& A_{1}\left(\nu D_{\zeta \zeta \zeta} v\right)=v_{0} D_{\zeta \zeta \zeta} v_{1}+v_{1} D_{\zeta \zeta \zeta} v_{0} \text {, } \\
& B_{1}\left(v D_{\zeta} v\right)=v_{0} D_{\zeta} v_{1}+v_{1} D_{\zeta} v_{0} \\
& A_{2}\left(v D_{\zeta \zeta \zeta} v\right)=v_{1} D_{\zeta \zeta \zeta} v_{2}+v_{1} D_{\zeta \zeta \zeta} v_{1}+v_{2} D_{\zeta \zeta \zeta} v_{0}, \\
& B_{2}\left(v D_{\zeta} v\right)=v_{1} D_{\zeta} v_{2}+v_{1} D_{\zeta} v_{1}+v_{2} D_{\zeta} v_{0} \\
& C_{0}\left(D_{\zeta} v D_{\zeta \zeta} v\right)=D_{\zeta} v_{0} D_{\zeta \zeta} v_{0} \\
& C_{1}\left(D_{\zeta} v D_{\zeta \zeta} v\right)=D_{\zeta} v_{0} D_{\zeta \zeta} v_{1}+D_{\zeta} v_{1} D_{\zeta \zeta} v_{0} \\
& C_{2}\left(D_{\zeta} v D_{\zeta \zeta} v\right)=D_{\zeta} v_{1} D_{\zeta \zeta} v_{2}+D_{\zeta} v_{1} D_{\zeta \zeta} v_{1}+D_{\zeta} v_{2} D_{\zeta \zeta} v_{0} \\
& v_{1}(\zeta, \mathfrak{J})=L^{-1}\left[\frac{\left(s^{\gamma}(1-\gamma)+\gamma\right)}{s^{\gamma}} L\left[D_{\zeta \zeta \mathfrak{\Im}} v_{0}-D_{\zeta} v_{0}+A_{0}-B_{0}+3 C_{0}\right]\right] \\
& =-\frac{1}{2} e^{(\zeta / 2)}\left((1-\gamma)+\frac{\gamma \mathfrak{J}^{\gamma}}{\Gamma(\gamma+1)}\right),
\end{aligned}
$$

for $\phi=2$,

$$
\begin{aligned}
& v_{2}(\zeta, \mathfrak{\Im})=L^{-1}\left[\frac{\left(s^{\gamma}(1-\gamma)+\gamma\right)}{s^{\gamma}} L\left[D_{\zeta \zeta \mathfrak{\Im}_{1}}-D_{\zeta} v_{1}+A_{1}-B_{1}+3 C_{1}\right]\right], \\
& \nu_{2}(\zeta, \mathfrak{I})=-\frac{1}{8} e^{(\zeta / 2)} \frac{\mathfrak{J}^{2 \gamma-1}}{\Gamma(2 \gamma)}+\frac{1}{4} e^{(\zeta / 2)}\left((1-\gamma)^{2}+\frac{\gamma^{2} \mathfrak{J}^{2 \gamma}}{\Gamma(2 \gamma+1)}+\frac{2(1-\gamma) \gamma \mathfrak{J}^{\gamma}}{\Gamma(\gamma+1)}\right)
\end{aligned}
$$

for $\phi=3$,

$$
\begin{aligned}
\nu_{3}(\zeta, \mathfrak{I})= & L^{-1}\left[\frac{\left(s^{\gamma}(1-\gamma)+\gamma\right)}{s^{\gamma}} L\left[D_{\zeta \zeta \mathfrak{J}_{2}}-D_{\zeta} \nu_{2}+A_{2}-B_{2}+3 C_{2}\right]\right], \\
v_{3}(\zeta, \mathfrak{J})= & -\frac{1}{32} e^{(\zeta / 2)} \frac{\mathfrak{J}^{3 \gamma-2}}{\Gamma(3 \gamma-1)}+\frac{1}{8} e^{(\zeta / 2)} \frac{\mathfrak{J}^{3 \gamma-1}}{\Gamma(3 \gamma)} \\
& -\frac{1}{8} e^{(\zeta / 2)}\left\{(1-\gamma)^{3}+\gamma(1-\gamma)\left(1+\gamma+2 \gamma^{2}\right) \frac{\mathfrak{J}^{\gamma}}{\Gamma(\gamma+1)}\right. \\
& \left.+\frac{3 \gamma^{2}(1-\gamma) \mathfrak{\Im}^{2 \gamma}}{\Gamma(2 \gamma+1)}+\frac{\gamma^{3} \Gamma(2 \gamma+1) \mathfrak{J}^{3 \gamma}}{\Gamma(3 \gamma+1)}\right\} .
\end{aligned}
$$

The modified decomposition method solution of example (1) is

$$
\begin{aligned}
\nu(\zeta, \mathfrak{I})= & e^{(\zeta / 2)}-\frac{1}{2} e^{(\zeta / 2)}\left((1-\gamma)+\frac{\gamma \mathfrak{J}^{\gamma}}{\Gamma(\gamma+1)}\right)-\frac{1}{8} e^{(\zeta / 2)} \frac{\mathfrak{J}^{2 \gamma-1}}{\Gamma(2 \gamma)} \\
& +\frac{1}{4} e^{(\zeta / 2)}\left((1-\gamma)^{2}+\frac{\gamma^{2} \mathfrak{\Im}^{2 \gamma}}{\Gamma(2 \gamma+1)}+\frac{2(1-\gamma) \gamma \mathfrak{J}^{\gamma}}{\Gamma(\gamma+1)}\right) \\
& -\frac{1}{32} e^{(\zeta / 2)} \frac{\mathfrak{J}^{3 \gamma-2}}{\Gamma(3 \gamma-1)}+\frac{1}{8} e^{(\zeta / 2)} \frac{\gamma^{3 \gamma-1}}{\Gamma(3 \gamma)}-\frac{1}{8} e^{(\zeta / 2)} \\
& \cdot\left\{(1-\gamma)^{3}+\gamma(1-\gamma)\left(1+\gamma+2 \gamma^{2}\right) \frac{\mathfrak{J}^{\gamma}}{\Gamma(\gamma+1)}\right. \\
& \left.+\frac{3 \gamma^{2}(1-\gamma) \mathfrak{J}^{2 \gamma}}{\Gamma(2 \gamma+1)}+\frac{\gamma^{3} \Gamma(2 \gamma+1) \mathfrak{J}^{3 \gamma}}{\Gamma(3 \gamma+1)}\right\}-\cdots .
\end{aligned}
$$

The simplification of equation (33)

$$
\begin{aligned}
\nu(\zeta, \mathfrak{I})= & e^{(\zeta / 2)}\left[1-2\left[(1-\gamma)+\frac{\gamma \mathfrak{J}^{\gamma}}{\Gamma(\gamma+1)}\right]-\frac{1}{8} \frac{\mathfrak{\Im}^{2 \gamma-1}}{\Gamma(2 \gamma)}\right. \\
& +\frac{1}{4}\left((1-\gamma)^{2}+\frac{\gamma^{2} \mathfrak{J}^{2 \gamma}}{\Gamma(2 \gamma+1)}+\frac{2(1-\gamma) \gamma \mathfrak{J}^{\gamma}}{\Gamma(\gamma+1)}\right) \\
& -\frac{1}{32} \frac{\mathfrak{J}^{3 \gamma-2}}{\Gamma(3 \gamma-1)}+\frac{1}{8} \frac{\mathfrak{\Im}^{3 \gamma-1}}{\Gamma(3 \gamma)} \\
& -\frac{1}{8}\left\{(1-\gamma)^{3}+\gamma(1-\gamma)\left(1+\gamma+2 \gamma^{2}\right) \frac{\mathfrak{J}^{\gamma}}{\Gamma(\gamma+1)}\right. \\
& \left.\left.+\frac{3 \gamma^{2}(1-\gamma) \mathfrak{J}^{2 \gamma}}{\Gamma(2 \gamma+1)}+\frac{\gamma^{3} \Gamma(2 \gamma+1) \mathfrak{J}^{3 \gamma}}{\Gamma(3 \gamma+1)}\right\}+\cdots\right] .
\end{aligned}
$$


Apply the variational method to obtain series form solution. The iteration formulas for equation (25), we get

$$
\begin{aligned}
v_{\phi+1}(\zeta, \mathfrak{I})= & v_{j}(\zeta, \mathfrak{\Im})-L^{-1}\left[\frac { ( s ^ { \gamma } ( 1 - \gamma ) + \gamma ) } { s ^ { \gamma } } L \left\{D_{\zeta \zeta \mathfrak{\Im} v_{\phi}+D_{\zeta} v_{\phi}}\right.\right. \\
& \left.\left.-v_{\phi} D_{\zeta \zeta v_{\phi}}+v_{\phi} D_{\zeta} v_{\phi}-3 D_{\zeta} v_{\phi} D_{\zeta \zeta} v_{\phi}\right\}\right],
\end{aligned}
$$

where

$$
v_{0}(\zeta, \mathfrak{J})=e^{(\zeta / 2)}
$$

For $\phi=0,1,2, \cdots$,

$$
\begin{aligned}
v_{1}(\zeta, \mathfrak{J})= & v_{0}(\zeta, \mathfrak{\Im})-L^{-1}\left[\frac { ( s ^ { \gamma } ( 1 - \gamma ) + \gamma ) } { s ^ { \gamma } } L \left\{D_{\zeta \zeta \Im} v_{0}+D_{\zeta} v_{0}\right.\right. \\
& \left.\left.-v_{0} D_{\zeta \zeta \zeta} v_{0}+v_{0} D_{\zeta} v_{0}-3 D_{\zeta} v_{0} D_{\zeta \zeta} v_{0}\right\}\right]
\end{aligned}
$$$$
v_{1}(\zeta, \mathfrak{I})=e^{(\zeta / 2)}-\frac{1}{2} e^{(\zeta / 2)}\left((1-\gamma)+\frac{\gamma \mathfrak{J}^{\gamma}}{\Gamma(\gamma+1)}\right)
$$$$
v_{2}(\zeta, \mathfrak{J})=v_{1}(\zeta, \mathfrak{J})-L^{-1}\left[\frac { ( s ^ { \gamma } ( 1 - \gamma ) + \gamma ) } { s ^ { \gamma } } L \left\{D_{\zeta \zeta \Im} \nu_{1}+D_{\zeta} \nu_{1}\right.\right.
$$$$
\left.\left.-v_{1} D_{\zeta \zeta \zeta} v_{1}+v_{1} D_{\zeta} v_{1}-3 D_{\zeta} v_{1} D_{\zeta \zeta} v_{1}\right\}\right]
$$$$
v_{2}(\zeta, \mathfrak{J})=e^{(\zeta / 2)}-\frac{1}{2} e^{(\zeta / 2)}\left((1-\gamma)+\frac{\gamma \mathfrak{J}^{\gamma}}{\Gamma(\gamma+1)}\right)-\frac{1}{8} e^{(\zeta / 2)} \frac{\mathfrak{I}^{2 \gamma-1}}{\Gamma(2 \gamma)}
$$$$
+\frac{1}{4} e^{(\zeta / 2)}\left((1-\gamma)^{2}+\frac{\gamma^{2} \mathfrak{\Im}^{2 \gamma}}{\Gamma(2 \gamma+1)}+\frac{2(1-\gamma) \gamma \mathfrak{\Im}^{\gamma}}{\Gamma(\gamma+1)}\right),
$$$$
v_{3}(\zeta, \mathfrak{J})=v_{2}(\zeta, \mathfrak{J})-L^{-1}\left[\frac { ( s ^ { \gamma } ( 1 - \gamma ) + \gamma ) } { s ^ { \gamma } } L \left\{D_{\zeta \zeta \mathcal{J}^{2}} \nu_{2}+D_{\zeta} \nu_{2}\right.\right.
$$$$
\left.\left.-v_{2} D_{\zeta \zeta \zeta} v_{2}+v_{2} D_{\zeta} v_{2}-3 D_{\zeta} v_{2} D_{\zeta \zeta} v_{2}\right\}\right]
$$

$$
\begin{aligned}
v_{3}(\zeta, \mathfrak{I})= & e^{(\zeta / 2)}-\frac{1}{2} e^{(\zeta / 2)}\left((1-\gamma)+\frac{\gamma \mathfrak{J}^{\gamma}}{\Gamma(\gamma+1)}\right)-\frac{1}{8} e^{(\zeta / 2)} \frac{\mathfrak{J}^{2 \gamma-1}}{\Gamma(2 \gamma)} \\
& +\frac{1}{4} e^{(\zeta / 2)}\left((1-\gamma)^{2}+\frac{\gamma^{2} \mathfrak{J}^{2 \gamma}}{\Gamma(2 \gamma+1)}+\frac{2(1-\gamma) \gamma \mathfrak{J}^{\gamma}}{\Gamma(\gamma+1)}\right) \\
& -\frac{1}{32} e^{(\zeta / 2)} \frac{\mathfrak{J}^{3 \gamma-2}}{\Gamma(3 \gamma-1)}+\frac{1}{8} e^{(\zeta / 2)} \frac{\mathfrak{J}^{3 \gamma-1}}{\Gamma(3 \gamma)}-\frac{1}{8} e^{(\zeta / 2)} \\
& \cdot\left\{(1-\gamma)^{3}+\gamma(1-\gamma)\left(1+\gamma+2 \gamma^{2}\right) \frac{\mathfrak{J}^{\gamma}}{\Gamma(\gamma+1)}\right. \\
& \left.+\frac{3 \gamma^{2}(1-\gamma) \mathfrak{J}^{2 \gamma}}{\Gamma(2 \gamma+1)}+\frac{\gamma^{3} \Gamma(2 \gamma+1) \mathfrak{J}^{3 \gamma}}{\Gamma(3 \gamma+1)}\right\},
\end{aligned}
$$

$$
\begin{aligned}
v(\zeta, \mathfrak{I})= & e^{(\zeta / 2)}-\frac{1}{2} e^{(\zeta / 2)}\left((1-\gamma)+\frac{\gamma \mathfrak{J}^{\gamma}}{\Gamma(\gamma+1)}\right)-\frac{1}{8} e^{(\zeta / 2)} \frac{\mathfrak{J}^{2 \gamma-1}}{\Gamma(2 \gamma)} \\
& +\frac{1}{4} e^{(\zeta / 2)}\left((1-\gamma)^{2}+\frac{\gamma^{2} \mathfrak{J}^{2 \gamma}}{\Gamma(2 \gamma+1)}+\frac{2(1-\gamma) \gamma \mathfrak{J}^{\gamma}}{\Gamma(\gamma+1)}\right) \\
& -\frac{1}{32} e^{(\zeta / 2)} \frac{\mathfrak{J}^{3 \gamma-2}}{\Gamma(3 \gamma-1)}+\frac{1}{8} e^{(\zeta / 2)} \frac{\gamma^{3 \gamma-1}}{\Gamma(3 \gamma)}-\frac{1}{8} e^{(\zeta / 2)} \\
& \cdot\left\{(1-\gamma)^{3}+\gamma(1-\gamma)\left(1+\gamma+2 \gamma^{2}\right) \frac{\mathfrak{J}^{\gamma}}{\Gamma(\gamma+1)}\right. \\
& \left.+\frac{3 \gamma^{2}(1-\gamma) \mathfrak{J}^{2 \gamma}}{\Gamma(2 \gamma+1)}+\frac{\gamma^{3} \Gamma(2 \gamma+1) \mathfrak{J}^{3 \gamma}}{\Gamma(3 \gamma+1)}\right\}-\cdots
\end{aligned}
$$

The exact solution of equation (25) at $\gamma=1$,

$$
v(\zeta, \mathfrak{J})=e^{((\zeta / 2)-(2 \Im / 3))} .
$$

In Figure 1, the analytical results of MDM/MVITM example 1 graphs show close contact with each other at $\gamma=1$ and 0.8 . It is investigated that analytical results are in close relation with the actual results of example 1. In Figure 2, the results of example 1 at different fractionalorder of the derivative are plotted at $\gamma=0.6$ and 0.4. Figure 3 shows the different fractional of two and three dimensional. The graphical representation has shown the convergence phenomena of fractional-order results towards the result at integer-order of example 1.

Example 8. Consider the time-fractional nonlinear Fornberg-Whitham equation is given as

$$
D_{\widetilde{\Im}}^{\gamma} \nu-D_{\zeta \zeta \Im^{\nu}} v+D_{\zeta} v=v D_{\zeta \zeta} \nu-v D_{\zeta} v+3 D_{\zeta} v D_{\zeta \zeta} \nu, \quad \mathfrak{J}>0,0<\gamma \leq 1 .
$$

The initial condition is

$$
v(\zeta, 0)=\cosh ^{2}\left(\frac{\zeta}{4}\right)
$$

Applying Laplace transformation of (39), we get

$$
\begin{aligned}
& \frac{s^{\gamma}}{\left(s^{\gamma}(1-\gamma)+\gamma\right)}\left\{L[v(\zeta, \Im)]-\frac{1}{s} v(\zeta, 0)\right\} \\
& \quad=L\left[D_{\zeta \zeta \Im} \nu-D_{\zeta} v+v D_{\zeta \zeta \zeta} v-v D_{\zeta} v+3 D_{\zeta} v D_{\zeta \zeta} v\right] .
\end{aligned}
$$

Using inverse Laplace transformation,

$$
\begin{aligned}
v(\zeta, \mathfrak{J})= & L^{-1}\left[\frac{v(\zeta, 0)}{s}-\frac{\left(s^{\gamma}(1-\gamma)+\gamma\right)}{s^{\gamma}} L\left\{D_{\zeta \zeta \Im} v-D_{\zeta} v\right.\right. \\
& \left.\left.+v D_{\zeta \zeta \zeta} v-v D_{\zeta} v+3 D_{\zeta} v D_{\zeta \zeta} v\right\}\right] .
\end{aligned}
$$



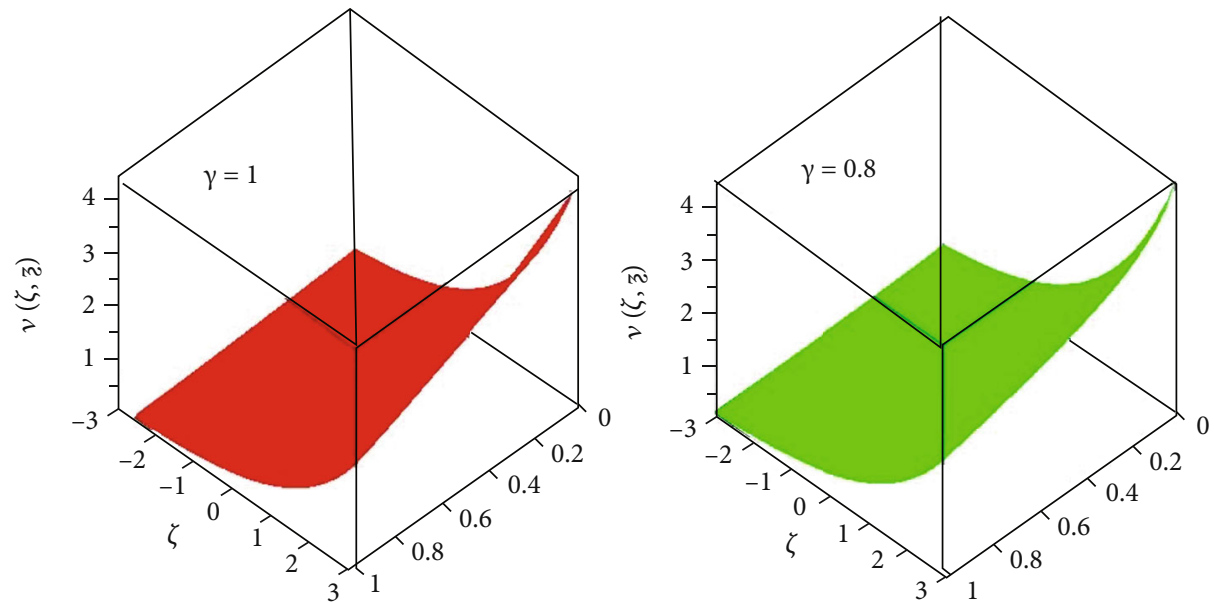

Figure 1: The solution graph of MDM/MVITM at $\gamma=1$ and 0.8 of example 1 .
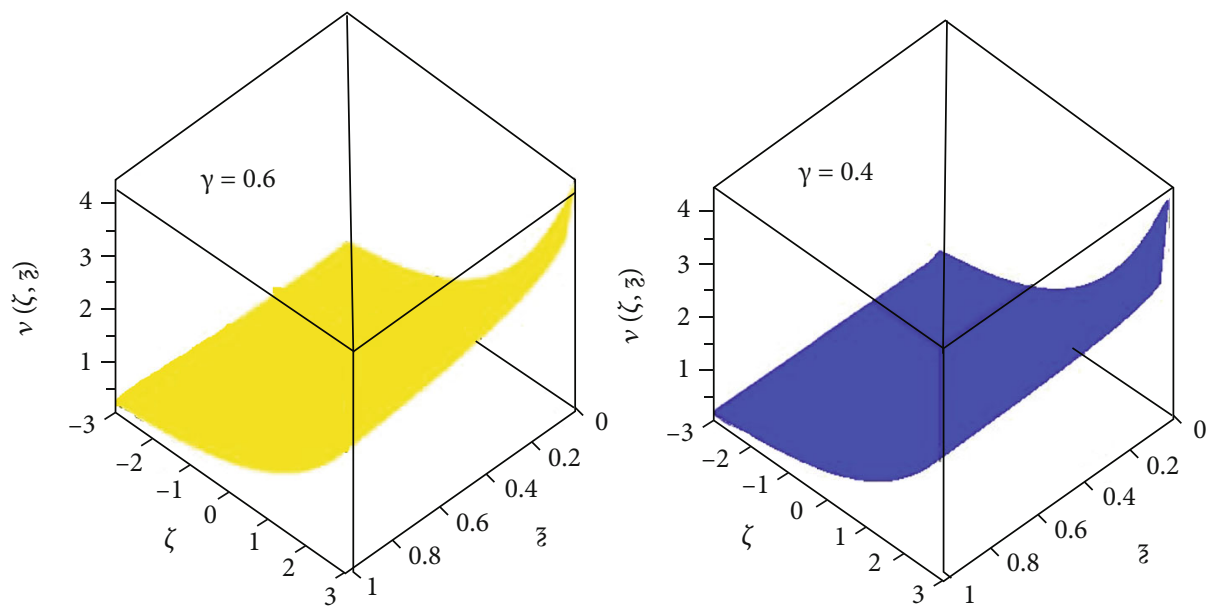

Figure 2: The solutions graph of MDM/MVITM at $\gamma=0.6$ and 0.4 of example 1 .
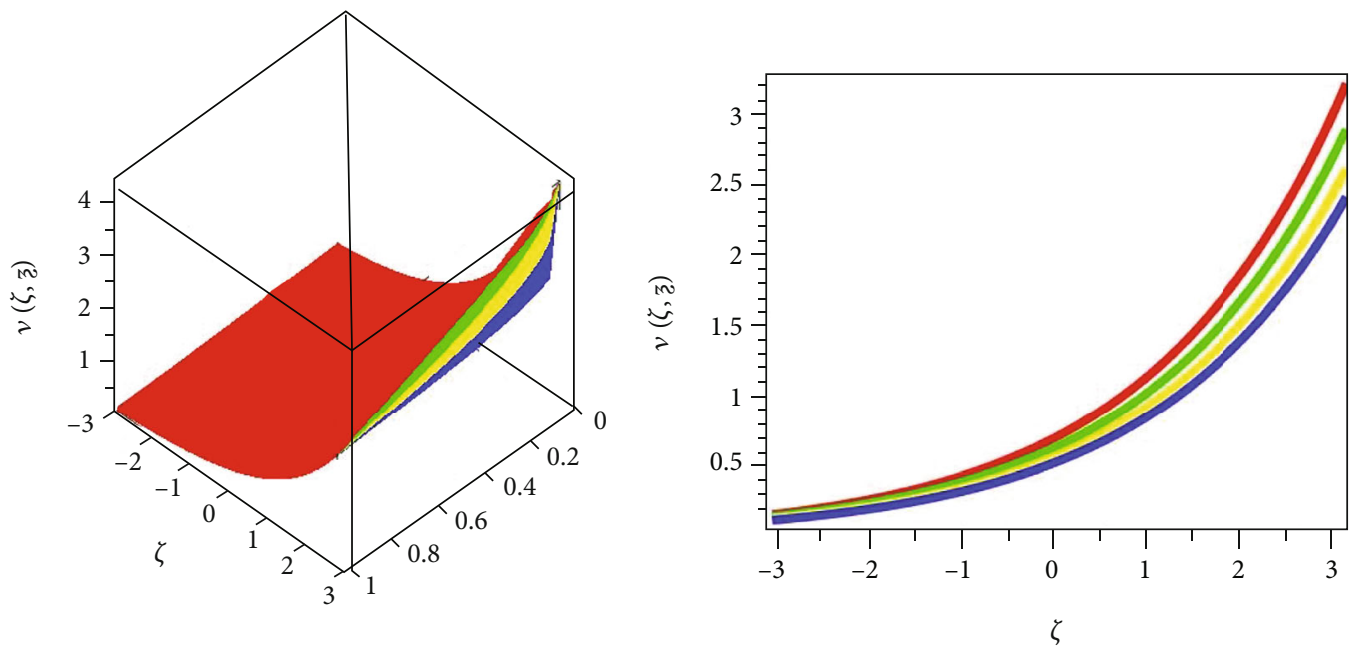

FIGURE 3: The different fractional-order graph of MDM/MVITM of example 1. 
Using ADM procedure, we get

$$
\begin{aligned}
& v_{0}(\zeta, \mathfrak{J})=L^{-1}\left[\frac{v(\zeta, 0)}{s}\right]=L^{-1}\left[\frac{\exp \left(\cosh ^{2}(\zeta / 4)\right)}{s}\right], \\
& v_{0}(\zeta, \Im)=\cosh ^{2}\left(\frac{\zeta}{4}\right)
\end{aligned}
$$

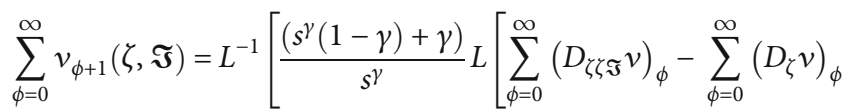

$$
\begin{aligned}
& \left.\left.+\sum_{\phi=0}^{\infty} A_{\phi}-\sum_{\phi=0}^{\infty} B_{\phi}+3 \sum_{\phi=0}^{\infty} C_{\phi}\right]\right], \quad \phi=0,1,2, \cdots,
\end{aligned}
$$

for $\phi=0$,

$$
\begin{aligned}
& v_{1}(\zeta, \mathfrak{\Im})=L^{-1}\left[\frac{\left(s^{\gamma}(1-\gamma)+\gamma\right)}{s^{\gamma}} L\left[D_{\zeta \zeta \mho^{2}} v_{0}-D_{\zeta} v_{0}+A_{0}-B_{0}+3 C_{0}\right]\right] \\
& =-\frac{11}{32} \sinh \left(\frac{\zeta}{4}\right)\left((1-\gamma)+\frac{\gamma \mathfrak{J}^{\gamma}}{\Gamma(\gamma+1)}\right),
\end{aligned}
$$

for $\phi=1$,

$$
\begin{aligned}
& v_{2}(\zeta, \mathfrak{\Im})=L^{-1}\left[\frac{\left(s^{\gamma}(1-\gamma)+\gamma\right)}{s^{\gamma}} L\left[D_{\zeta \zeta \Im} v_{1}-D_{\zeta} v_{1}+A_{1}-B_{1}+3 C_{1}\right]\right], v_{2}(\zeta, \mathfrak{\Im}) \\
& =-\frac{11}{28} \sinh \left(\frac{\zeta}{4}\right)\left((1-\gamma)+\frac{\gamma \Im^{\gamma}}{\Gamma(\gamma+1)}\right) \\
& +\frac{121}{1024} \cosh \left(\frac{\zeta}{4}\right)\left((1-\gamma)^{2}+\frac{\gamma^{2} \Im^{2 \gamma}}{\Gamma(2 \gamma+1)}+\frac{2(1-\gamma) \gamma \mathfrak{\Im}^{\gamma}}{\Gamma(\gamma+1)}\right),
\end{aligned}
$$

for $\phi=2$,

$$
\begin{aligned}
& v_{3}(\zeta, \Im)=L^{-1}\left[\frac{\left(s^{\gamma}(1-\gamma)+\gamma\right)}{s^{\gamma}} L\left[D_{\zeta \zeta \Im} v_{2}-D_{\zeta} v_{2}+A_{2}-B_{2}+3 C_{2}\right]\right], v_{3}(\zeta, \Im) \\
& =-\frac{11}{512} \sinh \left(\frac{\zeta}{4}\right)\left((1-\gamma)+\frac{\gamma \widetilde{\mathfrak{J}}^{\gamma}}{\Gamma(\gamma+1)}\right) \\
& +\frac{121}{2048} \cosh \left(\frac{\zeta}{4}\right)\left((1-\gamma)^{2}+\frac{\gamma^{2} \mathfrak{\Im}^{2 \gamma}}{\Gamma(2 \gamma+1)}+\frac{2(1-\gamma) \gamma \mathfrak{\Im}^{\gamma}}{\Gamma(\gamma+1)}\right) \\
& -\frac{1331}{49152} \sinh \left(\frac{\zeta}{4}\right)\left\{(1-\gamma)^{3}+\gamma(1-\gamma)\left(1+\gamma+2 \gamma^{2}\right) \frac{\mathfrak{J}^{\gamma}}{\Gamma(\gamma+1)}\right. \\
& \left.+\frac{3 \gamma^{2}(1-\gamma) \mathfrak{\Im}^{2 \gamma}}{\Gamma(2 \gamma+1)}+\frac{\gamma^{3} \Gamma(2 \gamma+1) \mathfrak{\Im}^{3 \gamma}}{\Gamma(3 \gamma+1)}\right\} \text {. }
\end{aligned}
$$

The MDM solution of example (8) is

$v(\zeta, \mathfrak{\Im})=v_{0}(\zeta, \mathfrak{\Im})+v_{1}(\zeta, \mathfrak{\Im})+v_{2}(\zeta, \mathfrak{\Im})+v_{3}(\zeta, \mathfrak{\Im})+v_{4}(\zeta, \mathfrak{\Im})+\cdots$,

$$
\begin{aligned}
v(\zeta, \mathfrak{\Im})= & \cosh ^{2}\left(\frac{\zeta}{4}\right)-\frac{11}{32} \sinh \left(\frac{\zeta}{4}\right)\left((1-\gamma)+\frac{\gamma \mathfrak{\Im}^{\gamma}}{\Gamma(\gamma+1)}\right) \\
& -\frac{11}{28} \sinh \left(\frac{\zeta}{4}\right)\left((1-\gamma)+\frac{\gamma \mathfrak{\Im}^{\gamma}}{\Gamma(\gamma+1)}\right) \\
& +\frac{121}{1024} \cosh \left(\frac{\zeta}{4}\right)\left((1-\gamma)^{2}+\frac{\gamma^{2} \mathfrak{\Im}^{2 \gamma}}{\Gamma(2 \gamma+1)}+\frac{2(1-\gamma) \gamma \mathfrak{J}^{\gamma}}{\Gamma(\gamma+1)}\right) \\
& -\frac{11}{512} \sinh \left(\frac{\zeta}{4}\right)\left((1-\gamma)+\frac{\gamma \mathfrak{J}^{\gamma}}{\Gamma(\gamma+1)}\right) \\
& +\frac{121}{2048} \cosh \left(\frac{\zeta}{4}\right)\left((1-\gamma)^{2}+\frac{\gamma^{2} \mathfrak{J}^{2 \gamma}}{\Gamma(2 \gamma+1)}+\frac{2(1-\gamma) \gamma \mathfrak{\Im}^{\gamma}}{\Gamma(\gamma+1)}\right) \\
& -\frac{1331}{49152} \sinh \left(\frac{\zeta}{4}\right)\left\{(1-\gamma)^{3}+\gamma(1-\gamma)\left(1+\gamma+2 \gamma^{2}\right) \frac{\mathfrak{\Im}^{\gamma}}{\Gamma(\gamma+1)}\right. \\
& \left.+\frac{3 \gamma^{2}(1-\gamma) \mathfrak{\Im}^{2 \gamma}}{\Gamma(2 \gamma+1)}+\frac{\gamma^{3} \Gamma(2 \gamma+1) \mathfrak{\Im}^{3 \gamma}}{\Gamma(3 \gamma+1)}\right\} \cdots
\end{aligned}
$$

Apply the variational method to find the analytical solution.

The iteration formulas for equation (39), we get

$$
\begin{aligned}
v_{\phi+1}(\zeta, \mathfrak{J})= & v_{j}(\zeta, \mathfrak{J})-L^{-1}\left[\frac { ( s ^ { \gamma } ( 1 - \gamma ) + \gamma ) } { s ^ { \gamma } } L \left\{D_{\zeta \zeta \Im} v_{\phi}+D_{\zeta} v_{\phi}\right.\right. \\
& \left.\left.-v_{\phi} D_{\zeta \zeta \zeta} v_{\phi}+v_{\phi} D_{\zeta} v_{\phi}-3 D_{\zeta} v_{\phi} D_{\zeta \zeta} v_{\phi}\right\}\right],
\end{aligned}
$$

where

$$
v_{0}(\zeta, \mathfrak{J})=\cosh ^{2}\left(\frac{\zeta}{4}\right)
$$

for $\phi=0,1,2, \cdots$

$$
\begin{aligned}
v_{1}(\zeta, \mathfrak{I})= & v_{0}(\zeta, \mathfrak{I})-L^{-1}\left[\frac { ( s ^ { \gamma } ( 1 - \gamma ) + \gamma ) } { s ^ { \gamma } } L \left\{D_{\zeta \zeta \Im} v_{0}+D_{\zeta} v_{0}\right.\right. \\
& \left.\left.-v_{0} D_{\zeta \zeta \zeta} v_{0}+v_{0} D_{\zeta} v_{0}-3 D_{\zeta} v_{0} D_{\zeta \zeta} v_{0}\right\}\right]
\end{aligned}
$$

$$
\begin{aligned}
v_{1}(\zeta, \mathfrak{I})= & \cosh ^{2}\left(\frac{\zeta}{4}\right)-\frac{11}{32} \sinh \left(\frac{\zeta}{4}\right)\left((1-\gamma)+\frac{\gamma \mathfrak{J}^{\gamma}}{\Gamma(\gamma+1)}\right) \\
v_{2}(\zeta, \mathfrak{I})= & v_{1}(\zeta, \mathfrak{\Im})-L^{-1}\left[\frac { ( s ^ { \gamma } ( 1 - \gamma ) + \gamma ) } { s ^ { \gamma } } L \left\{D_{\zeta \zeta \Im_{1} v_{1}+D_{\zeta} v_{1}}\right.\right. \\
& \left.\left.-v_{1} D_{\zeta \zeta \zeta} v_{1}+v_{1} D_{\zeta} v_{1}-3 D_{\zeta} v_{1} D_{\zeta \zeta} v_{1}\right\}\right],
\end{aligned}
$$

$v_{2}(\zeta, \mathfrak{I})=\cosh ^{2}(\zeta / 4)-11 / 32 \sinh (\zeta / 4)\left((1-\gamma)+\left(\gamma \mathfrak{\Im}^{\gamma}\right.\right.$ $/ \Gamma(\gamma+1)))-11 / 28 \sinh (\zeta / 4)\left((1-\gamma)+\left(\gamma \mathfrak{\Im}^{\gamma} / \Gamma(\gamma+1)\right)\right)+$ $121 / 1024 \cosh (\zeta / 4)\left((1-\gamma)^{2}+\left(\gamma^{2} \mathfrak{J}^{2 \gamma} / \Gamma(2 \gamma+1)\right)+(2(1-\right.$ $\left.\left.\gamma) \gamma \mathfrak{\Im}^{\gamma} / \Gamma(\gamma+1)\right)\right)$,

$$
v_{3}(\zeta, \mathfrak{\Im})=v_{2}
$$

$(\zeta, \mathfrak{J})-L^{-1}\left[\frac{\left(s^{\gamma}(1-\gamma)+\gamma\right)}{s^{\gamma}} L\left\{D_{\zeta \zeta \Im} \nu_{2}+D_{\zeta} \nu_{2}-v_{2} D \zeta \zeta \zeta \nu_{2}+v_{2}\right.\right.$ 

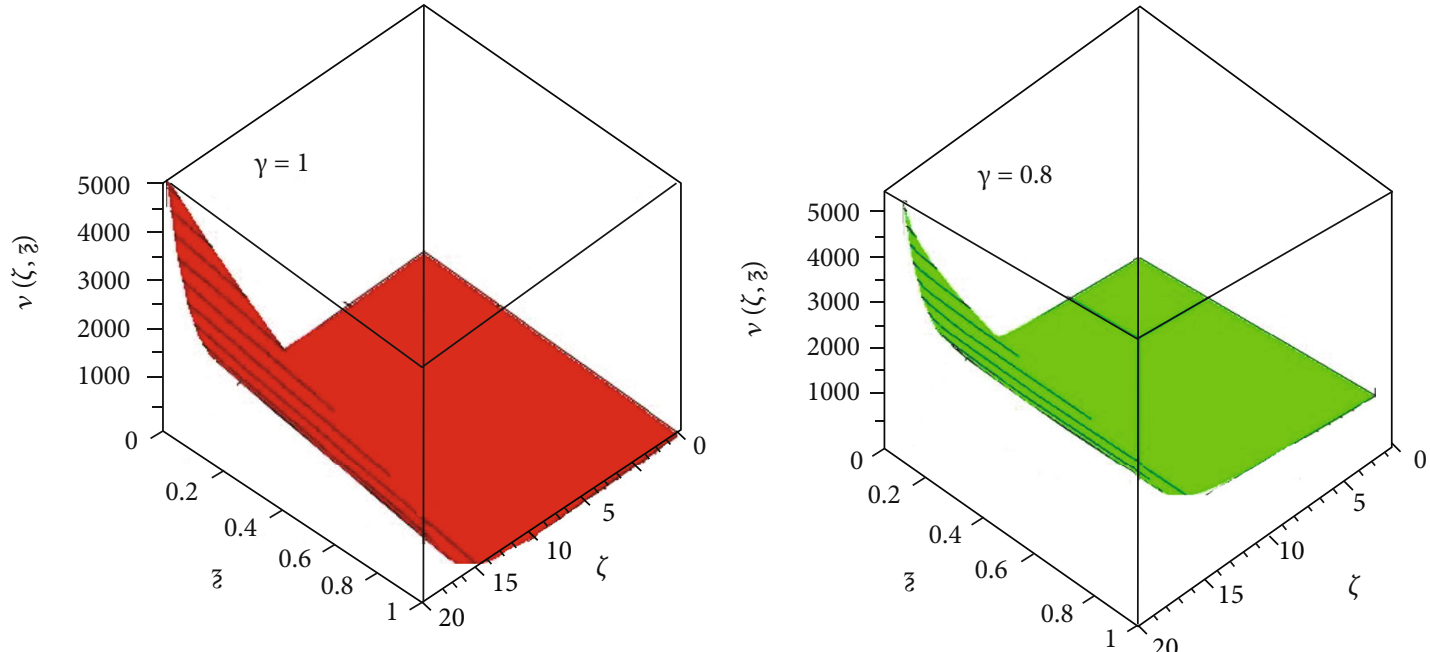

Figure 4: The solutions graph of MDM/MVITM at $\gamma=1$ and 0.8 of example 2.
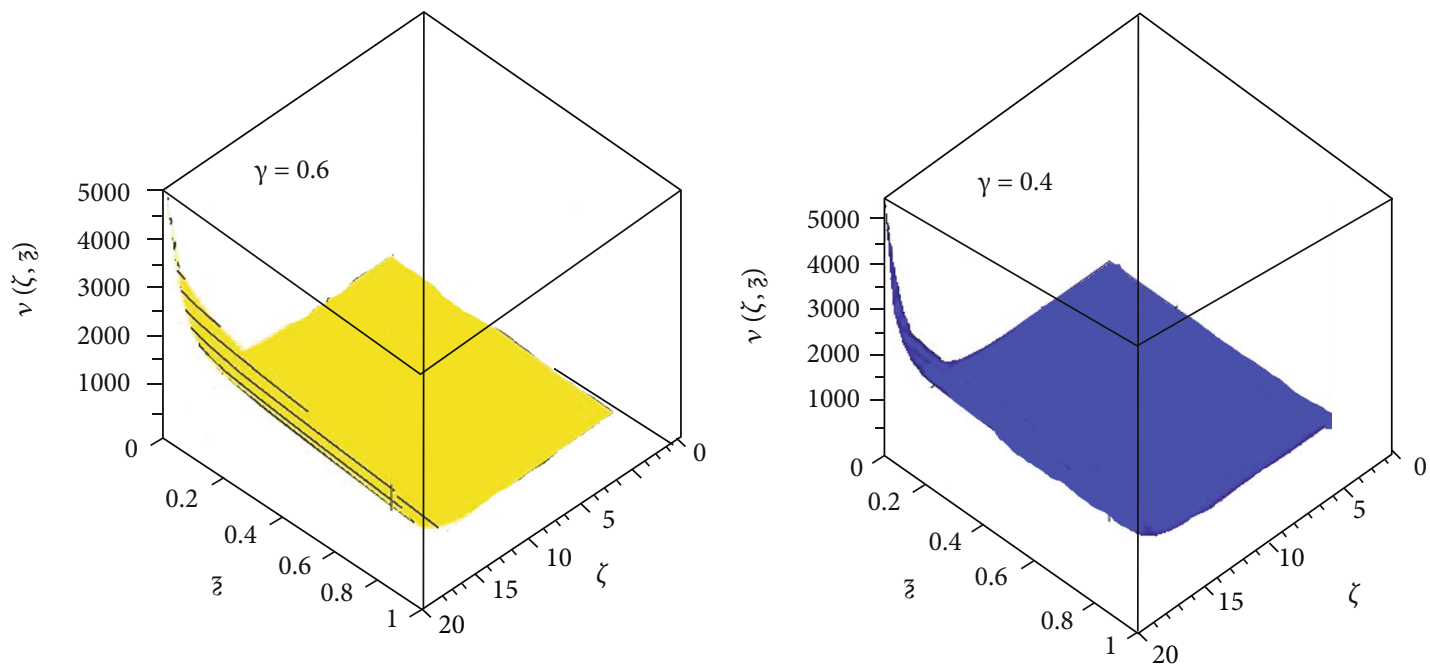

FIgURE 5: The solutions graph of MDM/MVITM at $\gamma=0.6$ and 0.4 of example 2 .

$\left.\left.D_{\zeta} v_{2}-3 \quad D_{\zeta} v_{2} D_{\zeta \zeta} v_{2}\right\}\right], v_{3} \quad(\zeta, \Im)=\cosh ^{2}\left(\frac{\zeta}{4}\right)-\frac{11}{32} \sinh \left(\frac{\zeta}{4}\right)((1$

$\left.-\gamma)+\frac{\gamma \mathfrak{\Im}^{\gamma}}{\Gamma(\gamma+1)}\right)-\frac{11}{28} \sinh \left(\frac{\zeta}{4}\right)\left((1-\gamma)+\frac{\gamma \mathfrak{\Im}^{\gamma}}{\Gamma(\gamma+1)}\right)+\frac{121}{1024} \cosh \left(\frac{\zeta}{4}\right.$ )$\left((1-\gamma)^{2}+\frac{\gamma^{2} \mathfrak{\Im}^{2 \gamma}}{\Gamma}(2 \gamma+1)+\frac{2(1-\gamma) \gamma \mathfrak{\Im}^{\gamma}}{\Gamma(\gamma+1)}\right)-\frac{11}{512} \sinh \left(\frac{\zeta}{4}\right)((1-\gamma$ )$\left.+\frac{\gamma \mathfrak{\Im}^{\gamma}}{\Gamma(\gamma+1)}\right)+\frac{121}{2048} \cosh \left(\frac{\zeta}{4}\right)\left((1-\gamma)^{2}+\frac{\gamma^{2} \mathfrak{\Im}^{2 \gamma}}{\Gamma}\right.$ $(2 \gamma+1)+$ $\left.\frac{2(1-\gamma) \gamma \mathfrak{\Im}^{\gamma}}{\Gamma(\gamma+1)}\right)-\frac{1331}{49152} \sinh \left(\frac{\zeta}{4}\right)\left\{(1-\gamma)^{3}+\gamma(1-\gamma) \quad\left(1+\gamma+2 \gamma^{2}\right)\right.$ $\frac{\mathfrak{J}^{\gamma}}{\Gamma(\gamma+1)}+\frac{3 \gamma^{2}(1-\gamma) \mathfrak{I}^{2 \gamma}}{\Gamma(2 \gamma+1)}+\gamma^{3} \Gamma(2 \gamma+1)$

$\left.\frac{\mathfrak{I}^{3 \gamma}}{\Gamma(3 \gamma+1)\}, v(\zeta, \mathfrak{I})=\cosh ^{2}\left(\frac{\zeta}{4}\right)-\frac{11}{32} \sinh \left(\frac{\zeta}{4}\right)\left((1-\gamma)+\frac{\gamma \widetilde{\Im} \gamma}{\Gamma}\right.}(\gamma+1)\right)-\frac{11}{28} \sinh$ $\left(\frac{\zeta}{4}\right)\left((1-\gamma)+\frac{\gamma \mathfrak{\Im}^{\gamma}}{\Gamma(\gamma+1)}\right)+\frac{121}{1024} \cosh \left(\frac{\zeta}{4}\right)\left((1-\gamma)^{2}+\frac{\gamma^{2} \mathfrak{\Im}^{2 \gamma}}{\Gamma(2 \gamma+1)}+\right.$ $\left.\frac{2(1-\gamma) \gamma \mathfrak{J}^{\gamma}}{\Gamma(\gamma+1)}\right)-\frac{11}{512} \sinh \left(\frac{\zeta}{4}\right)((1-\gamma)+\gamma \mathfrak{I}$

$\left.\frac{\gamma}{\Gamma(\gamma+1))+\frac{121}{2048} \cosh \left(\frac{\zeta}{4}\right)\left((1-\gamma)^{2}\right.}+\frac{\gamma^{2} \mathfrak{\Im}^{2 \gamma}}{\Gamma(2 \gamma+1)}+\frac{2(1-\gamma) \gamma \mathfrak{\Im}^{\gamma}}{\Gamma(\gamma+1)}\right)-\frac{1331}{49152} \sinh$ $\left(\frac{\zeta}{4}\right)\left\{(1-\gamma)^{3}+\gamma(1-\gamma)\left(1+\gamma+2 \gamma^{2}\right) \frac{\mathfrak{\Im}^{\gamma}}{\Gamma}\right.$ $\left.(\gamma+1)+\frac{3 \gamma^{2}(1-\gamma) \mathfrak{J}^{2 \gamma}}{\Gamma(2 \gamma+1)}+\frac{\gamma^{3} \Gamma(2 \gamma+1) \mathfrak{J}^{3 \gamma}}{\Gamma(3 \gamma+1)}\right\}+\cdots . \quad$ The exact solution of equation (39) at $\gamma=1$,

$$
v(\zeta, \mathfrak{J})=\cosh ^{2}\left(\frac{\zeta}{4}-\frac{11 \mathfrak{I}}{24}\right)
$$

In Figure 4, the analytical results of MDM/MVITM example 2 graphs show that close contact with each other at $\gamma=1$ and 0.8 . It is investigated that analytical results are in close relation with the actual results of example 2 . In Figure 5, the results of example 2 at different fractionalorder of the derivative are plotted at $\gamma=0.6$ and 0.4 . Figure 6 shows the different fractional of two and three dimensional. The graphical representation has shown the convergence phenomena of fractional-order results towards the result at integer-order of example 2. 

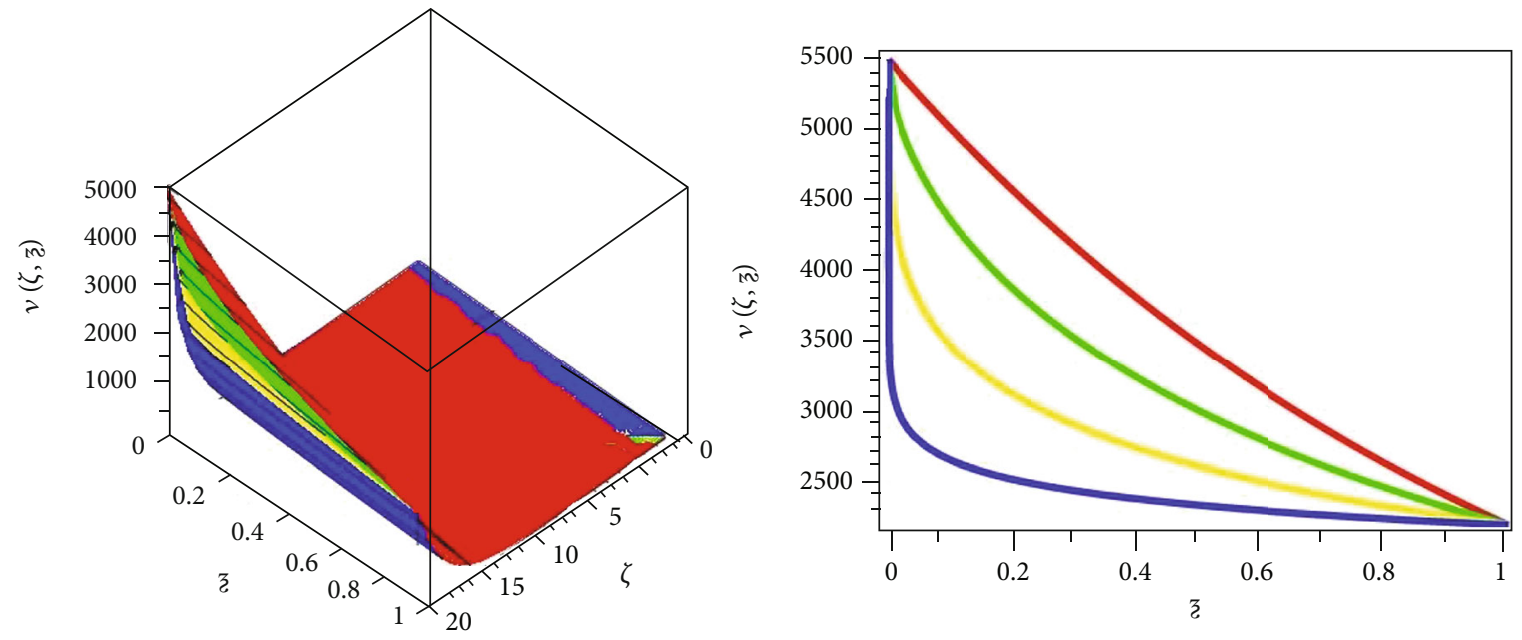

Figure 6: The different fractional-order graph of MDM/MVITM of example 2.

\section{Conclusion}

In this paper, we have been successfully applied two modified methods to investigate the approximate solutions of fractional Fornberg-Whitham equations. Agreement between numerical results obtained by the modified decomposition method and modified variational iteration method involving fractional-order derivatives with Mittag-Leffler kernel with exact result appears very appreciable by means of illustrative results in figures. The proposed techniques are easy to implement, effective, and suitable for achieving the results of nonlinear fractional Fornberg-Whitham equations. Moreover, both the modified decomposition method and variational iteration method provide the convergent series results with easily calculated components without applying any linearization, perturbation, or limiting assumptions. Finally, we can conclude the suggested methods are more accurate and highly methodical and which can be applied to investigate nonlinear models that arise in applied sciences.

\section{Data Availability}

The numerical data used to support the findings of this study are included within the article.

\section{Conflicts of Interest}

The authors declare that there are no conflicts of interest regarding the publication of this article.

\section{Acknowledgments}

This research has been funded by Scientific Research Deanship at University of Ha'il, Saudi Arabia, through project number RG-21 005.

\section{References}

[1] G. B. Whitham, "Variational methods and applications to water waves," Proceedings of the Royal Society of London. Series
A. Mathematical and Physical Sciences, vol. 299, no. 1456, pp. 6-25, 1967.

[2] B. Fornberg and G. B. Whitham, "A numerical and theoretical study of certain nonlinear wave phenomena. Philosophical Transactions of the Royal Society of London," Series A, Mathematical and Physical Sciences, vol. 289, no. 1361, pp. 373-404, 1978.

[3] K. S. Miller and B. Ross, An Introduction to the Fractional Calculus and Fractional Differential Equations, Wiley, 1993.

[4] J. Singh, D. Kumar, and J. J. Nieto, "A reliable algorithm for a local fractional tricomi equation arising in fractal transonic flow," Entropy, vol. 18, no. 6, p. 206, 2016.

[5] P. K. Gupta and M. Singh, "Homotopy perturbation method for fractional Fornberg-Whitham equation," Computers \& Mathematics with Applications, vol. 61, no. 2, pp. 250-254, 2011.

[6] A. A. Alderremy, H. Khan, R. Shah, S. Aly, and D. Baleanu, "The analytical analysis of time-fractional FornbergWhitham equations," Mathematics, vol. 8, no. 6, p. 987, 2020.

[7] P. Sunthrayuth, A. M. Zidan, S. W. Yao, R. Shah, and M. Inc, "The comparative study for solving fractional-order Fornberg-Whitham equation via $\rho$-Laplace transform," Symmetry, vol. 13, no. 5, p. 784, 2021.

[8] J. Singh, D. Kumar, and S. Kumar, "New treatment of fractional Fornberg-Whitham equation via Laplace transform," Ain Shams Engineering Journal, vol. 4, no. 3, pp. 557-562, 2013.

[9] M. Caputo and M. Fabrizio, "A new definition of fractional derivative without singular kernel," Progress in Fractional Differentiation and Applications, vol. 1, no. 2, pp. 1-13, 2016.

[10] M. Naeem, A. M. Zidan, K. Nonlaopon, M. I. Syam, Z. Al-Zhour, and R. Shah, "A new analysis of fractionalorder equal-width equations via novel techniques," Symmetry, vol. 13, no. 5, p. 886, 2021.

[11] J. Singh, D. Kumar, and J. J. Nieto, "Analysis of an El NinoSouthern Oscillation model with a new fractional derivative," Chaos, Solitons \& Fractals, vol. 99, pp. 109-115, 2017.

[12] R. P. Agarwal, F. Mofarreh, R. Shah, W. Luangboon, and K. Nonlaopon, "An analytical technique, based on natural transform to solve fractional-order parabolic equations," Entropy, vol. 23, no. 8, article e23081086, p. 1086, 2021. 
[13] A. Atangana and D. Baleanu, "New fractional derivatives with nonlocal and non-singular kernel: theory and application to heat transfer model," 2016, https://arxiv.org/abs/1602.03408.

[14] D. Kumar, J. Singh, D. Baleanu, and Sushila, "Analysis of regularized long-wave equation associated with a new fractional operator with Mittag-Leffler type kernel," Physica A: Statistical Mechanics and its Applications, vol. 492, pp. 155-167, 2018.

[15] J. Singh, D. Kumar, and D. Baleanu, "On the analysis of chemical kinetics system pertaining to a fractional derivative with Mittag-Leffler type kernel," Journal of Nonlinear Science, vol. 27, no. 10, article 103113, 2017.

[16] D. Baleanu, A. Jajarmi, and M. Hajipour, "A new formulation of the fractional optimal control problems involving MittagLeffler nonsingular kernel," Journal of Optimization Theory and Applications, vol. 175, no. 3, pp. 718-737, 2017.

[17] G. Adomian, Nonlinear Stochastic Operator Equations, Academic Press, 1986.

[18] G. Adomian, "Solution of physical problems by decomposition," Computers \& Mathematics with Applications, vol. 27, no. 9-10, pp. 145-154, 1994.

[19] H. Jafari, E. Tayyebi, S. Sadeghi, and C. M. Khalique, "A new modification of the Adomian decomposition method for nonlinear integral equations," The International Journal of Advances in Applied Mathematics and Mechanics, vol. 1, no. 4, pp. 33-39, 2014.

[20] D. Lesnic, "The decomposition method for forward and backward time-dependent problems," Journal of Computational and Applied Mathematics, vol. 147, no. 1, pp. 27-39, 2002.

[21] A. M. A. El-Sayed and M. Gaber, "The Adomian decomposition method for solving partial differential equations of fractal order in finite domains," Physics Letters A, vol. 359, no. 3, pp. 175-182, 2006.

[22] M. Ghoreishi, A. M. Ismail, and N. H. M. Ali, "Adomian decomposition method (ADM) for nonlinear wave-like equations with variable coefficient," Applied Mathematical Sciences, vol. 4 , no. $49,2010$.

[23] J. H. He, "Variational iteration method - a kind of non-linear analytical technique: some examples," International Journal of Non-Linear Mechanics, vol. 34, no. 4, pp. 699-708, 1999.

[24] J. H. He, "Approximate analytical solution for seepage flow with fractional derivatives in porous media," Computer Methods in Applied Mechanics and Engineering, vol. 167, no. 1-2, pp. 57-68, 1998.

[25] J. H. He, "Some asymptotic methods for strongly nonlinear equations," International Journal of Modern Physics B, vol. 20, no. 10, pp. 1141-1199, 2006.

[26] J. H. He and X. H. Wu, "Variational iteration method: new development and applications," Computers \& Mathematics with Applications, vol. 54, no. 7-8, pp. 881-894, 2007.

[27] D. D. Ganji and A. Sadighi, "Application of homotopyperturbation and variational iteration methods to nonlinear heat transfer and porous media equations," Journal of Computational and Applied Mathematics, vol. 207, no. 1, pp. 24-34, 2007.

[28] T. Ozis and A. Yildirim, "Traveling wave solution of Korteweg-de Vries equation using He's homotopy perturbation method," The International Journal of Nonlinear Sciences and Numerical Simulation, vol. 8, pp. 239-242, 2007.

[29] M. A. Noor and S. T. Mohyud-Din, "Variational iteration method for solving higher-order nonlinear boundary value problems using He's polynomials," International Journal of
Nonlinear Sciences and Numerical Simulation, vol. 9, no. 2, pp. 141-156, 2008.

[30] I. Ali, H. Khan, R. Shah, D. Baleanu, P. Kumam, and M. Arif, "Fractional view analysis of acoustic wave equations, using fractional-order differential equations," Applied Sciences, vol. 10, no. 2, p. 610, 2020.

[31] V. F. Morales-Delgado, J. F. Gomez-Aguilar, S. Kumar, and M. A. Taneco-Hernandez, "Analytical solutions of the KellerSegel chemotaxis model involving fractional operators without singular kernel," The European Physical Journal Plus, vol. 133, no. 5 , p. 200,2018 . 